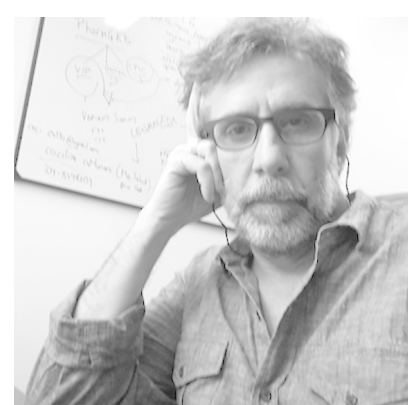

Hernán Dopazo

Dr. Ciencias Biológicas. CONICET / FCEYN-UBA hdopazo@ege.fcen.uba.ar

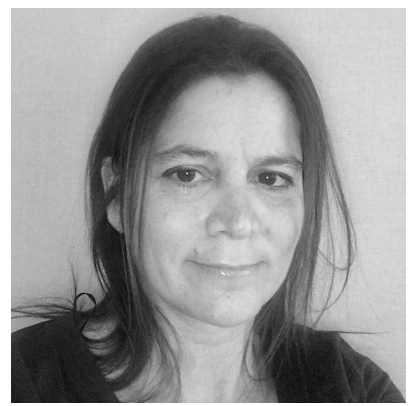

\section{Mariana Berenstein \\ Dra en Ciencias Químicas, KNOWHUB - CHILE mberenstein@knowhub.cl}

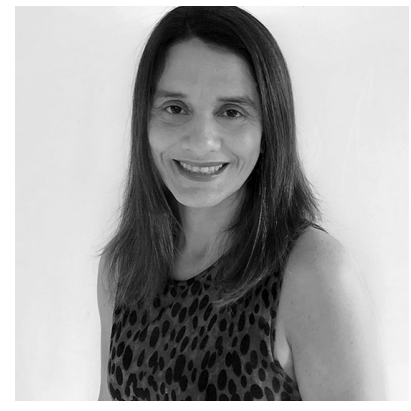

Andrea S. Llera

Dra. en Bioquímica.

FIL - CONICET

a_llera@yahoo.com

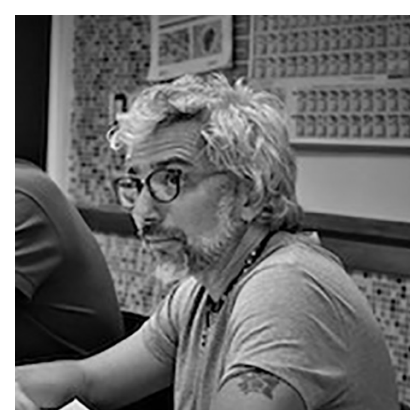

Rolando Gonzáles-José

Dr. Ciencias Biológicas. CENPAT - CONICET

rolando@cenpat-conicet.gob.ar

\title{
Genomas, enfermedades y medicina de precisión: un Proyecto Nacional
}

Resumen: La medicina de precisión se presenta en los países centrales como un nuevo paradigma en el tratamiento de algunas enfermedades. Basada en el conocimiento del genoma de cada individuo, se sustenta en el principio de que el éxito en el tratamiento de las enfermedades, y la respuesta a los fármacos, depende del conocimiento detallado de las variantes genéticas de los individuos, los porcentajes de ancestría, del medio ambiente y de otros factores que no pueden ser extrapolados de forma directa entre poblaciones. Nuestro país debe decidir si importa de otros países este conocimiento para aplicarlo de manera inespecífica, o pone en marcha un proyecto nacional para beneficiar de manera inclusiva a nuestra población con los adelantos propios de la biomedicina y la salud del siglo XXI.

Palabras claves: Medicina de Precisión, Genómica, Poblaciones Humanas, Salud.

\section{Genomes, Diseases and Precision Medicine: a National Project}

Abstract: Precision medicine is presented in the central countries as a new paradigm in the treatment of some diseases. Based on the knowledge of the genome of each individual, it is based on the principle that the success in the treatment of diseases, and the response to drugs, depends on the detailed knowledge of the genetic variants of the individuals, the percentages of ancestry, of the environment and other factors that can not be directly extrapolated among populations. Our country must decide whether to take this knowledge from other countries to apply it in an unspecified way, or launch a national project to benefit our population inclusively with the advances of biomedicine and health in the 21st century. Key words: Precision Medicine, Genomics, Human Populations, Health 
| Ciencia, Tecnología y Política | Año 2 | N² | Enero-Junio 2019 | ISSN 2618-3188 | www.revistas.unlp.edu.ar/CTyP |

\section{Genomas, enfermidades e medicina de precisão: um Projeto Nacional}

Resumo: A medicina de precisão é apresentada nos países centrais como um novo paradigma no tratamento de algumas doenças. Com base no conhecimento do genoma de cada indivíduo, fundamenta-se no princípio de que o sucesso no tratamento de doenças e a resposta às drogas dependem do conhecimento detalhado das variantes genéticas dos indivíduos, das porcentagens de ancestralidade, do meio ambiente e outros fatores que não podem ser diretamente extrapolados entre as populações. Nosso país deve decidir se importa esse conhecimento de outros países para aplicá-lo de maneira não especifica, ou lançar um projeto nacional para beneficiar nossa população, com os avanços da biomedicina e da saúde no século XXI. Palabras claves: Medicina de Precisão, Genômica, Populações Humanas, Saúde

\section{El Genoma humano típico}

"El progreso de la ciencia depende de nuevas técnicas, nuevos descubrimientos y nuevas ideas, posiblemente en ese orden". La frase de Sydney Brenner, Premio Nobel de Medicina 2002, caracteriza el cambio de paradigma de la biomedicina de los últimos 30 años. En ese tiempo, hemos pasado del estudio minucioso de unas pocas proteínas en diferentes rutas metabólicas, al estudio completo de miles de productos génicos y sus interacciones en la célula. El catalizador inicial de esta transformación fue el Proyecto Genoma Humano (PGH) ${ }^{1}$ el cual, ciertamente, no fue consecuencia de un descubrimiento trascendente, ni proporcionó una idea revolucionaria sobre nuestra visión del mundo, sino que consistió en la aplicación de conocimientos ya consolidados y la puesta a punto de tecnologías y procesos seriales encadenados, similares a los de una fábrica, para producir datos genómicos de forma masiva y generar la primera referencia del genoma de nuestra especie. Este es un buen ejemplo de cómo la combinación de los conocimientos ya existentes, con enfoques nuevos, pueden generar nuevos nichos científicos con aplicaciones directas a las necesidades de una sociedad. El objetivo a corto plazo del PGH, parece hoy muy modesto: producir un mapa de referencia que nos permitiera localizar con exactitud la totalidad de elementos que componen el genoma humano. A largo plazo, no obstante, el objetivo sería mucho más ambicioso y complejo: conocer todos los factores genéticos que nos predisponen a desarrollar enfermedades, y comprender su funcionamiento. A partir de allí, se propuso avanzar en un nuevo programa de la medicina basado en la información del genoma de cada paciente y en el conocimiento de cómo los genes interactúan con el ambiente. Esto es lo que se denomina medicina de precisión.

Para avanzar en este nuevo programa se requería de nuevos proyectos e iniciativas que, entre otras cosas, desarrollaran tecnologías que aceleraran y disminuyeran los costos de producción de los datos genómicos; generaran información sobre la variabilidad genética de las poblaciones; construyeran herramientas informáticas que garantizaran el acceso de esta información a la comunidad científica; definieran nuevos estándares de análisis genético y discutieran los alcances éticos, legales y sociales de sus resultados.

Entre estas iniciativas, el proyecto denominado 1.000 Genomas $^{2}$ analizó la variabilidad genética humana en más de 2.500 individuos de 26 poblaciones en distintos continentes. Una de las conclusiones principales de este proyecto es que no existe el genoma humano normal, sino el genoma humano típico (más

${ }^{1} \mathrm{PGH}$. goo.gl/ko3GIL

2 1KGP. www.internationalgenome.org 
frecuente), el cual difiere del genoma humano de referencia realizado por el PGH, en aproximadamente 4 a 5 millones de variantes genéticas. La representación latinoamericana en este consorcio, encargado de catalogar el acervo genético de la humanidad, se restringió a unos pocos individuos de México, Puerto Rico, Colombia y Perú.

\section{Variantes genéticas y medicina de precisión}

¿Por qué el estudio de las enfermedades necesita del análisis de la variabilidad genética humana? Las variantes genéticas representan en el genoma lo mismo que los carteles de señalización de una carretera: son indicadores que permiten determinar el riesgo de padecer una enfermedad y necesitamos conocerlos para poder luchar contra ella. De manera formal, las necesitamos para realizar estudios estadísticos de asociación que muestren una relación significativa entre una variante genética y una enfermedad. Para lograr esto se realizan estudios de asociación de genoma completo (o GWAS, por sus siglas en inglés)3). Estos estudios comparan una a una las centenares de miles de variantes genéticas en grupos de individuos sanos y enfermos (casos y controles, respectivamente; considerando que la estructura de edad, sexo, ancestría genética y estilo de vida sea lo más homogénea posible entre ambos grupos). Si una de estas variantes es significativamente más frecuente en el grupo de casos, estamos en presencia de una variante genética que incrementa el riesgo de contraer la enfermedad. Por ejemplo, se ha comprobado que en individuos de ascendencia europea, aquellos que contienen en su genoma una variante genética específica tienen entre 2 y 4 veces más riesgo de desarrollar Alzheimer a edad avanzada que el promedio de la población.

El descubrimiento de estas variantes genéticas asociadas a una enfermedad (biomarcadores) es el primer paso para el desarrollo de la medicina de precisión ya que muchas decisiones sobre salud y enfermedad pueden tomarse conociendo las características genéticas del paciente; por ejemplo, saber que una persona tiene, a nivel genético, una susceptibilidad aumentada a padecer un determinado cáncer puede generar una conducta preventiva más estricta (dieta, ejercicio, frecuencia de tamizaje). También la prescripción de medicamentos hoy en día es guiada por biomarcadores que demuestran si el paciente es candidato a responder o no a una determinada terapia, o a tener más o menos efectos secundarios. Según la Coalición para la Medicina de Precisión (PMC, por sus siglas en inglés)4 , desde 2005 hasta 2017 el número de medicamentos aprobados con un biomarcador asociado se incrementó desde el 5\% hasta el 34\% en EE.UU. y la tendencia es que este porcentaje se incremente aún más en los próximos años. Surge entonces la pregunta ¿los estudios de GWAS realizados principalmente con genomas europeos, sirven para identificar el riesgo de enfermedades en poblaciones de cualquier otro continente, por ejemplo Latinoamérica?

\section{Sesgos entre poblaciones}

La principal base de datos de GWAS contiene más de 3.200 estudios, de los cuales el 81\% son de población europea. Los estudios con hispanos y latinoamericanos (0.5\%) se encuentran por debajo de los

\footnotetext{
${ }^{3}$ Genome-Wide Association Studies - GWAS Catalog: https://www.ebi.ac.uk/gwas/

${ }^{4}$ PMC. www.personalizedmedicinecoalition.org/
} 
africanos (1\%), y estos están muy lejos de los asiáticos (14\%). Los datos muestran asociaciones muy significativas con enfermedades humanas complejas, con rasgos que son factores de riesgo de enfermedades, y con respuestas a fármacos. Pero el $80 \%$ de todas estas asociaciones corresponde a estudios con genomas europeos exclusivamente (Bustamante, et al., 2011; Popeyoy \& Fulleton, 2016; Sirugo, eta al. 2019). Este sesgo, impuesto por la práctica científica, tiene razones históricas, económicas y políticas obvias, pero resulta dramático si consideramos que más de tres cuartas partes de la población del mundo vive en África, Asia y Latinoamérica.

Este problema no es sólo de las bases de datos de GWAS, sino también de las que catalogan la variabilidad genética de otro tipo de enfermedades, como fibrosis quística, hemofilia, Frágil X, Tay-Sachs, Huntington, etc., en su mayoría causadas por mutaciones en un único gen, con nula o muy escasa influencia del medio ambiente.

\section{Política científica y programas de Medicina de Precisión}

La descripción de los sesgos poblacionales arriba mencionados hace evidente que los avances en materia de variabilidad genética humana y medicina de precisión tienen la capacidad de producir una enorme desigualdad potencial entre los habitantes de los países ricos que financian proyectos relevantes de biomedicina en favor de sus habitantes, y los países pobres con instituciones que parecieran apostar al "que investiguen otros", con la vana ilusión de importar el conocimiento sin advertir que esta no es la solución. Desde hace más de 7 años, diferentes investigadores están alertando al mundo sobre este problema (Hindorff, et al., 2018; Ledford, 2019; Mills \& Rahal, 2019,). Se han propuesto ideas y planes para superar este futuro escenario de desigualdad, como la financiación de proyectos que incluyan poblaciones minoritarias y diversas, la colaboración entre instituciones financiadoras de diferentes países, y por supuesto, la promoción de la financiación nacional para el desarrollo de programas de largo alcance en genómica poblacional y medicina de precisión. Es en este escenario complejo, dinámico, y atravesado por fuertes intereses financieros ligados a la producción de nuevos fármacos y métodos genómicos de diagnóstico, donde los sistemas científicos y de salud pública de los países periféricos no pueden permanecer ajenos. Esto cobra mayor relevancia cuando en dicho país (y Argentina es un ejemplo de ello), su historia pasada y reciente han preconfigurado un escenario de mestizaje masivo, sumamente diverso e ininterrumpido desde épocas del primer poblamiento americano hasta la actualidad, ya que cualquier desarrollo corre el riesgo de no ser inclusivo para todos los habitantes del país. Un ejemplo bien estudiado de este problema es el de cáncer de mama en mujeres latinoamericanas, publicado recientemente (Zavala, et al., 2019).

Es la propia contingencia histórica de nuestro continente en general, y de nuestro país en particular, la que nos obliga a reflexionar con inteligencia estratégica acerca de cómo posicionar las capacidades técnicas, financieras y humanas ante este campo de investigación y desarrollo que indefectiblemente habrá que abordar y consolidar en un futuro cercano. En este sentido, es importante observar y analizar cómo se están desarrollando los programas de medicina de precisión en países que comenzaron prematuramente a trabajar en esa dirección.

Entre las principales iniciativas actuales de largo alcance en medicina de precisión se encuentra UK Bio- 
bank ${ }^{5}$, una sociedad anónima e institución de beneficencia del Reino Unido creada en 2006 con financiación del sistema nacional de salud, el gobierno central y el Wellcome Trust. Entre 2006 y 2010, UK Biobank reclutó aproximadamente 500.000 voluntarios adultos que serán seguidos por el sistema de salud durante 30 años. El objetivo es mejorar la prevención, el diagnóstico y el tratamiento de una amplia gama de enfermedades graves y potencialmente mortales, como cáncer, enfermedades cardíacas, derrames cerebrales, diabetes, artritis, osteoporosis, trastornos oculares, depresión y distintas formas de demencia. Los voluntarios completaron un cuestionario sobre estilo de vida, historia clínica, hábitos nutricionales, peso, altura, presión arterial, etc., a los cuales se agregan muestras de sangre, orina, ADN y últimamente imágenes de alta definición. Durante la duración del estudio, el servicio de salud nacional centraliza los eventos de enfermedades, prescripción de medicamentos y muertes de los participantes. Desde marzo de 2012 este conjunto de datos comenzó a estar disponible para ser solicitado por científicos de todo el mundo, ya sean del sector público o privado, de la industria, la academia u organizaciones benéficas. Una de las obligaciones es que los investigadores que utilicen estos datos deben devolver sus hallazgos a UK Biobank; esto está regulado por el Acuerdo de Transferencia de Materiales o Datos que los investigadores que acceden a los mismos deben cumplimentar.

El objetivo general de este tipo de iniciativas es, en resumidas cuentas, cambiar por completo el ecosistema de la investigación biomédica de un país, poniendo a disposición los datos a un número grande de investigadores de manera centralizada y regulada, y evitando el escenario habitual de distribuir recursos para lograr objetivos muchas veces repetidos de forma individual en laboratorios independientes. En 2015, el UK Biobank liberó el primer conjunto de datos genéticos correspondientes a 150 mil participantes. Dos años más tarde, ese número de voluntarios ascendió a 500 mil. Sólo una semana después de liberar esos datos, se logró duplicar la cantidad de marcadores ligados a enfermedades, volcando esa información en un navegador web para facilitar la investigación por parte de otros especialistas. Al día de hoy, alrededor de 7.000 investigadores se han registrado en el UK Biobank como usuarios del biobanco, con más de 1.400 proyectos y cerca de 600 artículos ya publicados (Kaiser \& Gibbons, 2019). En el corto plazo, se podrá contar con grandes tamaños muestrales, que permitirán utilizar la información de múltiples marcadores genéticos para realizar mejores y más precisas estimaciones de factores de riesgo poligénicos (aquellos influenciados por más de un gen, Martin et al. 2019). Esta escala de datos no se puede obtener con grupos de investigación aislados, sino que requieren iniciativas de escala nacional.

UK Biobank, sin embargo, también tiene limitaciones. La más importante es que el $94 \%$ de sus datos son de individuos europeos. No están representados, individuos asiáticos, de descendencia africana o americanos nativos por lo que la utilidad de los hallazgos del biobanco para individuos de otros lugares del mundo es una incógnita. Los investigadores que dirigen el proyecto confían en que otros países proveerán datos de poblaciones más diversas; por ejemplo, el proyecto "All of Us" de EEUU, planea llegar al millón de individuos, y ha prometido aportar la mitad de minorías de origen afroamericano,

${ }^{5}$ UK BIOBANK. www.ukbiobank.ac.uk/ 
hispánicos y asiáticos, entre otros.

Resulta evidente entonces que, independientemente del tipo de variación genética que intervenga en la determinación de una enfermedad y del estado actual o futuro de las bases de datos, los países que quieran garantizar un sistema de salud beneficioso y equitativo para sus ciudadanos deben intervenir de forma efectiva para generar biobancos que colecten muestras biológicas. Deberían realizar además un seguimiento con colección periódica de nuevos datos, descubrir variantes genéticas novedosas, registrar los diferentes estilos de vida de sus ciudadanos en relación a factores de riesgo como tabaquismo, educación y acceso a la salud y colaborar en proyectos de envergadura con otros países del mundo en beneficio de todos.

\section{PoblAR: un Programa Nacional de Medicina de Precisión}

En 2013 la Dirección de Vinculación Tecnológica del CONICET (actualmente Gerencia de Vinculación Tecnológica), detectó que en la órbita del CONICET y las Universidades Nacionales diversos grupos de investigación con fuertes lazos internacionales participaban activamente, pero en forma aislada, en el estudio genético de las poblaciones humanas y el desarrollo de biobancos para investigación. Además de la baja articulación científica, se detectó que estos grupos no cooperaban para obtener financiamiento. El diagnóstico general fue que los grupos locales podían ser vistos, en mayor o menor medida, como proyectos de "asistencia" a iniciativas internacionales. Ante este panorama, teniendo en cuenta la fuerte sinergia potencial que existía entre las iniciativas en términos de investigación biomédica de base poblacional, y luego de conversaciones con los representantes locales de esos consorcios internacionales, la DVT-CONICET decidió organizar el Primer Workshop Argentino de Genómica Médica y Poblacional, que se realizó en el Centro Nacional Patagónico en julio del 2014. Este evento nucleó por primera vez, a diversos grupos de investigación del CONICET, el Ministerio de Salud de la Nación y las Universidades Nacionales, y permitió identificar las complejidades y potencialidades de una articulación a mayor escala. En ese mismo año se creó el consorcio PoblAR ${ }^{6}$, una iniciativa con el objetivo de avanzar de forma efectiva hacia la aplicación de la medicina de precisión. En mayo de 2015 se realizó el Segundo Workshop Argentino de Genómica Médica y Poblacional, celebrado en el Museo Argentino de Ciencias Naturales "Bernardino Rivadavia" y en octubre de 2015 el Tercer Workshop Argentino de Genómica Médica y Poblacional, celebrado en Polo Científico Tecnológico.

El trabajo intenso y articulado entre más de 40 investigadores, becarios y técnicos permitió que las instituciones avanzaran en la firma de una Carta de Intención entre CONICET, la ANLIS (Administración Nacional de Laboratorios e Institutos de Salud) y las Universidades Nacionales de Córdoba, Jujuy y Misiones, en noviembre 2015ㄱ, en la que las partes se comprometen a impulsar la creación de

\footnotetext{
${ }^{6}$ Consorcio PobIAR. Integrantes e Instituciones (orden alfabético): Alfaro E; Aquilano E; Argüelles C; Avena S; Bailliet G; Beltramo J; Berenstein M; Bravi C; Carnese R; Cuello M; Dejean C; Demarchi D; Dipierri J; Dopazo, H; García A; Gonzáles José R; Guichón R; Llera A; Jurado Medina L; Miretti M; Motti J; Parolin ML; Pauro M; Paz P; Ramallo, V; Rodríguez G; Rudzinski M; Santos MR; Schwab M; Silvero M. CONICET, CENPAT, FFyH-UNC, FIL, UBA, UCAMI, U. Maimónides, UNC, UNCPBA, UNaM, UNJU.

7 https://goo.gl/yJcKqD
} 
PobIAR: un Centro de Referencia y Biobanco Nacional.

Una vez alcanzado este primer hito de institucionalización, e identificada en los sucesivos encuentros una estructura de "nodos" de muestreo y análisis, se llevó adelante una Reunión de Nodos, en noviembre de 2016, y una presentación ante autoridades el 2 de noviembre de ese mismo año. Esta consolidación de la iniciativa resultó atractiva para algunos biobancos ya establecidos, como fue el caso del Instituto de Efectividad Clínica y Sanitaria (IECS), que a través de dos convenios, cedió e incorporó muestras y equipamientos a la iniciativa PobIAr (enero y junio de 2017).

\section{Características y objetivos de PoblAR}

PobIAR es una iniciativa que se propone crear un centro de referencia encargado de diseñar, poner en marcha y consolidar el muestreo de bioespecímenes (diferentes tipos de material biológico de distintos individuos) y datos asociados de la población argentina para conformar un biobanco de datos abiertos y de referencia para cualquier investigación genómica biomédica considerada prioritaria para nuestro país. Uno de sus objetivos es el estudio de la composición de ancestrías de nuestra población, la cual es más diversa y compleja que la clásica reportada de sólo tres componentes: Europeo, Nativo-Americano y Africano. Recientemente, los que integran PobIAR han deducido que, para casi 100 argentinos distribuidos desde Jujuy hasta Tierra del Fuego y desde Buenos Aires hasta Mendoza, existen en todos ellos, al menos 3 linajes nativo-americanos, los provenientes del: 1- Norte Andino, 2- Centro y Sur Patagónico y 3- Noreste o Región del Gran Chaco (Luisi, et al., 2018). La importancia de este hallazgo radica en la ausencia de toda referencia sobre los dos últimos componentes de ancestría en las bases de datos internacionales, con las consecuencias que esto tiene sobre la interpretación de las variantes genéticas y la asociación a enfermedades.

PoblAR se propone, en el mediano plazo, constituir un foco de interés para el sector público y privado involucrado en el desarrollo de fármacos y terapias de medicina de precisión con base genómica. Entre sus funciones rutinarias contempla: la diagramación y puesta en marcha de muestreos sobre nuestra población, la coordinación y control del acceso a los datos por parte de especialistas del sector público y/o privado, y el fomento de la investigación biomédica basada en los datos genómicos y sus variables asociadas.

Hasta el momento, no ha sido posible organizar en nuestro país grandes bases de datos nacionales que combinen la epidemiología molecular con la tradicional, ya sea de población clínica y/o normal, de acceso controlado a distintos niveles (incluyendo datos de libre acceso), con estándares de calidad comprobados y con un número suficiente de muestras que permita abordar estudios como los que requiere la medicina de precisión. Sin embargo, el grado de desarrollo en materia de recursos físicos y humanos de nuestro sistema científico tecnológico y de salud es suficiente para la creación de un Centro que coordine una plataforma de muestreo multicéntrico, multidisciplinario y permanente que permita incluir un Biobanco para la investigación biomédica en nuestro país.

La creación y manejo de este tipo de bases de datos requiere un esfuerzo de coordinación humana, logística, financiera e institucional que favorezca y potencie los análisis a gran escala, que precisan necesariamente grandes muestras y una asociación precisa entre el dato genómico, el fenotípico y el ambiental.

Una lección aprendida de experiencias similares (como la mencionada más arriba, UK Biobank) es que las 
iniciativas de gran escala no son sólo proyectos de investigación que incrementan su complejidad en el tiempo, sino que requieren de una aproximación diferente que incorpore la gestión institucional, y una visión de "procesos", además del rigor científico.

La existencia de un banco que combine metadatos genómicos y no-genómicos que refleje en su lógica y diseño de muestreo la diversidad poblacional de nuestro país, generará un abaratamiento de costos e independencia científica para el desarrollo de estos productos biomédicos. La institucionalización del banco, por otro lado, permitirá un mejor control estatal del acceso a los datos y su posterior uso, tanto por parte del sector público como del privado. En el largo plazo, esta iniciativa cuenta con externalidades muy importantes. Por un lado, será un interlocutor importante en materia de provisión de datos e información para el sector farmacéutico. En este sentido, el Estado contará con soberanía en la toma de decisiones a la hora de proveer la información de base a uno u otro sector, dependiendo de la coyuntura y las políticas de desarrollo del sector. Por otro lado, esta iniciativa se verá complementada y potenciada cuando se implementen en todo el territorio sistemas de historia clínica digitales, que permitan contar con información sistematizada, masiva y digital acerca de antecedentes de enfermedades y otros metadatos de interés que permitan orientar pulsos de muestreo específicos.

\section{Estado actual de PoblAr}

El recorrido institucional descripto arriba, así como la firma de acuerdos y convenios en torno a esta iniciativa indica que el interés por ella trascendió a los investigadores involucrados, y escaló a niveles de respaldo institucional. El objetivo general de PobIAR ha sido planteado tanto por la vía ejecutiva (en reuniones con los ex-Ministerios de Salud y Ciencia, Tecnología e Innovación Productiva) como por la vía legislativa (proyecto de Ley presentado en el Senado).

No obstante, las autoridades de las instituciones intervinientes no han podido concretar la creación del Centro, avance que ya contaba con suficiente consenso de todas las partes. Ya sea por falta de fondos, por falta de voluntad política, o una combinación de ambos factores, existe un congelamiento total de la iniciativa desde junio de 2017. Ante esta situación los investigadores participantes han decidido continuar trabajando (independientemente de las decisiones de las instituciones) en torno a la unificación de protocolos de toma de datos en distintas regiones del país, en analizar preliminarmente aquellos datos compartidos informalmente entre grupos de investigación, y en iniciar el camino legislativo en torno a la sanción de un Proyecto de Ley que de creación al Centro de Referencia y Biobanco Poblar. El proyecto de ley en cuestión fue ingresado para su tratamiento en el Senado de la Nación a fines de 2018, desde el despacho de la Senadora Nancy Gonzalez (UC-Chubut), estando hasta el momento a la espera de su tratamiento en las comisiones de Presupuesto, Salud y Ciencia y Tecnología.

\section{Conclusiones}

La medicina de precisión no ha desarrollado todo su potencial, y parecería limitarse a ensayos genómicos promovidos por la industria farmacéutica para definir una terapia dirigida de muy alto costo. Sin embargo, esta visión deja de lado la combinación de la información genómica con la proveniente del medio ambiente para la búsqueda de soluciones en distinto grupos de enfermedades. En Latinoamérica, la deficiencia de los 
registros de salud pública es evidente, y es por ello que no sabemos lo suficiente, ni de genómica ni de epidemiología de enfermedades crónicas en nuestra población. La creación de un Centro de Referencia y Biobanco Nacional basada en un paradigma de investigación y desarrollo, debería verse como un primer paso necesario para obtener una imagen lo más certera y federal posible de las características de la población argentina en lo que respecta a factores de riesgo, tanto genómicos como ambientales. De esta manera, y con estos datos, la salud pública argentina obtendría evidencias para rediseñar políticas de salud pública que evalúen la conveniencia de aplicar acciones basadas en medicina de precisión, no ya en el diagnóstico y tratamiento, sino en la prevención de enfermedades crónicas.

La implementación de la medicina de precisión en nuestro país en particular, y en la región en general, debe ser entendida desde la perspectiva de las políticas públicas en salud. Como en otros casos donde la innovación científica y tecnológica ha determinado el dominio de cadenas de valor relativamente independientes de la fluctuación del valor de las commodities, esta alteración virtuosa de los mercados se origina en fuertes decisiones políticas que involucran al Estado como inversor de riesgo, al menos en las fases iniciales del proceso (Dvorkin, E. 2017). La producción de reactores, la industria satelital, la producción de aluminio, la fabricación de radares o instrumental para medicina nuclear son sólo algunos ejemplos de ello en nuestro país, pero también un indicador de cómo las estrategias que han resultado efectivas a la hora de lograr desarrollo basado en innovación son de largo plazo, alto riesgo, y fuerte presencia del Estado. Dado el escenario actual, en donde las innovaciones en materia de salud están en manos de actores privados, de no acometer el sector público una política de I+D clara en el campo de la medicina de precisión, todo indica que las terapias y los medicamentos que irrumpan en el mercado no estarán al alcance de toda la población. La existencia de un biobanco de referencia de datos genómicos y ambientales que analicen la diversidad fundamentalmente mestiza de nuestro país, es el primer paso para un plan nacional de largo alcance en medicina de precisión en favor de todos los argentinos.

\section{Bibliografía}

Bustamante C., Burchard E., \& F. De la Vega 2011. Genomics for the world. Nature 475 (7355):163-5.

Dvorkin, E. (2017) ¿Qué ciencia quiere el país? Los estilos tecnológicos y los proyectos nacionales. Ed. Colihue. I.S.B.N : 9789876843089

Hindorff LA., Bonham V., Brody L., Ginoza M., Hutter C., Manolio T. \& E. Green. 2018. Prioritizing diversity in human genomics research. Nat Rev Genet. 9(3): 175-185.

Kaiser, J. \& Gibbons, A. 2019. Biology in the bank. Science 363,18-20.

Ledford, H. (2019). Cancer geneticists tackle troubling ethnic bias in studies. Nature 568, 154-155.

Luisi P, Berros JM, Consorcio PobIAR, Ramallo, V \& H. Dopazo. 2018. Fine-scale human genetic structure reveals Patagonian, Andean, and Gran-Chaco ancestry components throughout Argentina. Congreso conjunto de la International Society of Computational Biology - Latin America (ISCG-LA) y la Iberoamerican Society for Bioinformatics (SOIBIO) - EMBnet 2018. Nov 5-9, 2018, Viña del Mar, Chile. 
Manrai AK, Funke B., Rehm H., Olesen M., Maron B., Szolovits P., Margulies D, Loscalzo J. \& I. Kohane. 2016. Genetic Misdiagnoses and the Potential for Health Disparities. New Eng. Jour. of Medicine 375: 655-665.

Martin, A. R., Kanai, M., Kamatani, Y., Okada, Y., Neale, B. M., \& Daly, M. J. 2019. Clinical use of current polygenic risk scores may exacerbate health disparities. Nature Genetics 51(4), 584-591.

Mills, M. C., \& Rahal, C. (2019). A scientometric review of genome-wide association studies. Communications Biology (2) 9, 1-11.

Popejoy A., \& S. Fullerton. 2016. Genomics is failing on diversity. Nature 538 (7624):161-164.

Sirugo, G., Williams, S. \& Tishkoff, S. (2019) The Missing Diversity in Human Genetic Studies. Cell 177, 26-31.

Zavala, V., et al. 2019. Genetic Epidemiology of Breast Cancer in Latin America. Genes, 10(2), 153-24. 